\title{
Hypercomplex Gel Changes to Organic/Inorganic Solid Solution by Phase Transition in an Artificial Biomineralization System
}

\author{
By Takashi IWATSUBO* and Tomohiko YAMAGUCHI
}

In an artificial biomineralization system, a polymer complex consisting of poly(vinyl alcohol) (PVA) and poly(acrylic acid) (PAA) was utilized as a mineralization field. The polymer complex was immersed in salt solution containing $x \mathrm{mM} \mathrm{CaCl}_{2}$, $0.6 x \mathrm{mM} \mathrm{KH} \mathrm{PO}_{4}$, and PAA at $\mathrm{pH}=7.4$. After equilibrium was reached, the weight uptake in the polymer complex was determined in the dry state. When $x$ was less than the transition concentration $\left(x_{\mathrm{T}}\right)$, the polymer complex was transformed into a hydrogel, and the dry weight uptake gradually increased with increasing $x$. On the other hand, at $x$ greater than $x_{\mathrm{T}}$, the polymer complex was transformed into an organic/inorganic solid composite, and the dry weight uptake was almost independent of $x$. The hydrogel formed was a hypercomplex gel composed of $\mathrm{Ca}^{2+}$ and $\mathrm{HPO}_{4}{ }^{2-}$ ions and the polymer complex held together by hydrogen bonds and electrostatic interactions. The organic/inorganic solid composite appeared to be a solid solution of the polymer complex and hydroxyapatite, as indicated by experiments on the saturation state of the salt solutions. Our results indicate that the hypercomplex gel changed discontinuously to the organic/inorganic solid solution at $x=x_{\mathrm{T}}$; this discontinuity represents a phase transition of hypercomplex gel to organic/inorganic solid solution that is an open system whose border is permeable to both energy and mass. The hypercomplex gel and the organic/inorganic solid solution can serve as models for the fundamental structures of cartilaginous and skeletal material respectively.

KEY WORDS: Biomineralization / Hypercomplex Gel / Organic/inorganic Solid Solution / Miscibility / Phase Transition /

Catilaginous Material / Skeletal Material /

Skeletal materials (endoskeleton and exoskeleton) formed by biomineralization are not pure $\mathrm{Ca}_{5}\left(\mathrm{PO}_{4}\right)_{3}(\mathrm{OH}), \mathrm{CaCO}_{3}$, or $\mathrm{SiO}_{2}$ but composites of organic polymers and the respective minerals. The polymers play a principal role in biomineralization and are believed to endow skeletal materials with resistance to fractures by overcoming the fragility inherent in the mineral component. Biomineralization is controlled by both species-specific genetic information and physicochemical parameters. Once the necessary solute ions and organic polymers have been produced and assembled by the organism, the subsequent stages of biomineralization are controlled primarily by physicochemical factors such as $\mathrm{pH}$ and the concentrations of the polymers and ions, which affect interactions between them in aqueous solution. Although the physicochemical mechanism of biomineralization has been investigated for many years, the mechanism is still unknown, even for cases in which the monomer sequence of the polymers is clarified.

In previous work, we discovered the physicochemical regularity in the polymer systems related to hydroxyapatite (HAP), $\mathrm{CaCO}_{3}$ and $\mathrm{SiO}_{2}$ skeletal materials, and an artificial biomineralization system has been constructed using a polymer complex hydrogel composed of poly(vinyl alcohol) (PVA) and poly(acrylic acid) (PAA) that is swollen in an aqueous solution containing PAA at $\mathrm{pH}=7.4{ }^{1}$ There is an attractive force between PVA and PAA through hydrogen bonding because the $-\mathrm{OH}$ of PVA acts as a proton donner while the $-\mathrm{COO}^{-}$acts as a proton acceptor. Since both PVA and PAA are water soluble, a covalent bond between PVA and PAA is introduced by partial esterification to prevent dissolution of the hydrogel. In this system, PVA serves as an analogue of bone collagen, which is rich in hydroxyproline residues, ${ }^{2}$ because both PVA and collagen are rich in $-\mathrm{OH}$ groups and hence can act as proton donors in hydrogen bonds. The PAA immobilized with PVA in the hydrogel serves as an analogue of bone sialoprotein $^{3}$ and other acidic proteins that are contained in bone and considered to be helpful in HAP mineralization. The similarity between PAA, bone sialoprotein and other acidic proteins is their acidic groups, which allow them to act as proton acceptors in hydrogen bonds. The PAA in the outer solution also serves as an analogue of osteopontin, osteocalcin ${ }^{3}$ and other acidic proteins which like PAA, bear acidic groups that are dissociated at $\mathrm{pH}=7.4$. Since this dissociation decreases the local activity product of dissolved ions by an electrostatic effect, ${ }^{4}$ PAA, osteopontin, osteocalcin and other acidic proteins can raise source ion concentrations of precipitation threshold in the outer solution. The similarities between collagen and PVA and also between bone sialoprotein and PAA imply that like PVA and PAA, collagen and bone sialoprotein can be expected to experience attractive forces due to hydrogen bonding and to form a polymer complex that is transformed into a hydrogel in aqueous solution.

From the viewpoint of constituent polymers, our artificial biomineralization system is also analogous to biomineralization systems involving $\mathrm{CaCO}_{3}$ skeletal material. Chitin is an insoluble polymeric component of the exoskeleton of crusta-

Nanotechnology Research Institute, National Institute of Advanced Industrial Science and Technology, Central 5, 1-1-1 Higashi, Tsukuba 305-8565, Japan

*To whom correspondence should be addressed (Tel: +81-29-861-4758, Fax: +81-29-861-6236, E-mail: t-iwatsubo@aist.go.jp). 
ceans and has $-\mathrm{NH}$ groups that act as proton donors in hydrogen bonds. Soluble acidic proteins such as crustcalcin ${ }^{5}$ and $\mathrm{CAP}-1^{6}$ extracted from exoskeletons are considered to be helpful in $\mathrm{CaCO}_{3}$ mineralization. Owing to the presence of acidic residues, these proteins are proton acceptors and hence may form hydrogen bonds with insoluble chitin to produce hydrogels in solution. CAP-1 also raises precipitation threshold concentrations of source ions for mineralization of $\mathrm{CaCO}_{3}$ in weakly alkaline solution. Our model system is also analogous to biomineralization systems for $\mathrm{SiO}_{2}$ skeletal material. The glycoprotein contained in diatom silica ${ }^{7}$ may serve as a proton donor, and the acidic polymer silaffin $-2^{8}$ is a proton acceptor that can combine with the glycoprotein and form a hydrogel in solution. Since silaffin-1 is a cationic polymer, ${ }^{9}$ it will dissociate and therefore raises threshold concentrations of source ions for mineralization of $\mathrm{SiO}_{2}$ in outer solutions at acidic $\mathrm{pH}$.

In our artificial biomineralization system, immersion of the PVA/PAA polymer complex in a solution of $\mathrm{CaCl}_{2}$, $\left(\mathrm{NH}_{4}\right)_{2} \mathrm{HPO}_{4}$, and PAA affords a polymer complex hydrogel that can be transformed into a transparent, dense organic/ inorganic solid composite. The peaks in the X-ray powder diffraction pattern of this composite material are located at Bragg angles similar to those of HAP but are broader than those of HAP. ${ }^{10}$ In our previous study, we measured the distribution of $\mathrm{Ca}, \mathrm{C}, \mathrm{O}$, and $\mathrm{P}$ within the composite material at the various stages of composite formation by means of energydispersive analysis of X-ray (EDX). ${ }^{1}$ In the intermediate stages of composite formation, the hypercomplex gel phase containing $\mathrm{HPO}_{4}{ }^{2-}$ and the organic/inorganic solid phase coexist. This solid phase grows at the cost of the gel phase. ${ }^{1}$ This twophase structure observed in the intermediate stages strongly implies that the change from hypercomplex gel phase to the solid phase represents a phase transition. In this study, we investigated the discontinuity of weight uptake measured in the equilibrium state by varying the salt concentration in the outer solution. We verify that this discontinuity means a phase transition from a hypercomplex gel to an organic/inorganic solid solution. On the basis of recognition of this phase transition system, we will discuss the structures and characteristics of various cartilaginous and skeletal materials.

\section{EXPERIMENTALS}

\section{Materials}

PVA (degree of polymerization, ca. 2000), $\mathrm{CaCl}_{2} \cdot 2 \mathrm{H}_{2} \mathrm{O}$, $\mathrm{NaCl}, \mathrm{KH}_{2} \mathrm{PO}_{4}, \mathrm{~K}_{2} \mathrm{HPO}_{4}$, and $1 \mathrm{M}$ tris(hydroxymethyl)aminomethane hydrochloride (Tris- $\mathrm{HCl}$ ) buffer were purchased from Wako Pure Chemical Industries (Osaka, Japan). Two types of PAA (MW $\sim 2000 \mathrm{Da}$ and average MW $250000 \mathrm{Da}$ ) were purchased from Sigma-Aldrich Corporation (St. Louis, MO, USA). Hydroxyapatite pellet CELLYARD ${ }^{\mathrm{TM}}$ was purchased from Pentax Corporation (Tokyo, Japan).

\section{Mineralization Conditions}

To prepare PVA/PAA complex films, aqueous solutions containing $1 \mathrm{wt} \%$ of PVA and $1 \mathrm{wt} \%$ of PAA (MW $250000 \mathrm{Da})$ were mixed at a PVA:PAA $(\mathrm{w} / \mathrm{w})$ ratio of $1: 1$ and stirred for $2 \mathrm{~h}$ at room temperature so that a homogeneous polymer solution was formed. The solution was cast onto polystyrene petri dishes. Water was allowed to evaporate at room temperature for almost one week, and then the films were dried in a vacuum at room temperature for $1 \mathrm{~d}$. The resulting films could easily be peeled off the dishes and were about $30 \mu \mathrm{m}$ thick and transparent, which indicates the good miscibility of PVA and PAA. The PVA/PAA complex films were heated at $115^{\circ} \mathrm{C}$ in a digitally regulated vacuum oven for $1 \mathrm{~h}$ to introduce cross-links by covalent bonds between the polymer chains of PVA and PAA by partial esterification. ${ }^{11}$ Circular pieces (radius $1 \mathrm{~cm}$ ) were cut from the film for experiments.

The salt solutions were $x \mathrm{mM}$ in $\mathrm{CaCl}_{2}, 0.6 x \mathrm{mM}$ in $\mathrm{KH}_{2} \mathrm{PO}_{4}$ $(0 \leq x \leq 5)$ both as the source ions for HAP, $0.139 \mathrm{mM}$ in the repeating unit of PAA (MW $2000 \mathrm{Da}$ ), and $140 \mathrm{mM}$ in $\mathrm{NaCl}$. The $\mathrm{pH}$ of the solution was maintained at 7.4 with $\mathrm{Tris}-\mathrm{HCl}$ buffer. The $\mathrm{NaCl}$ concentration and $\mathrm{pH}$ values were chosen because they were similar to those of body fluid. ${ }^{12}$ The high concentrations of source ions in the solution were maintained by the presence of free PAA. In the absence of PAA, precipitation occurred, whereas in the presence of PAA, the solution remained transparent for more than 2 weeks even at $x=5$. The PAA in the outer solution clearly raised precipitation threshold in our system. The salt solution was transparent at $x=5.5$, whereas precipitated at $x=6.0$, which indicates that $5.5<x_{\mathrm{P}}<6.0$, where $x_{\mathrm{P}}$ is the precipitation threshold. The polymer films were soaked in $200 \mathrm{~mL}$ of the salt solution prepared in a glass beaker, which was covered with sealing film and thermostated at $30^{\circ} \mathrm{C}$. The salt solution was renewed to keep the salt concentration as constant as possible.

To determine whether the solution was supersaturated, we used simplified simulated body fluid ${ }^{12}$ (s-SBF) as a positive control; the s-SBF was composed of $2.5 \mathrm{mM} \mathrm{CaCl}_{2}, 1.0 \mathrm{mM}$ $\mathrm{K}_{2} \mathrm{HPO}_{4}$, and $140 \mathrm{mM} \mathrm{NaCl}$ and had a $\mathrm{pH}$ of 7.4 by Tris- $\mathrm{HCl}$ buffer.

\section{Characterization of Composites}

After an appropriate time intervals and renewals of solution, the soaked film was removed from the solution and rinsed three times in pure water for $10 \mathrm{~min}$ each to remove residual soluble ions from the composite. The composite was then dried for $1 \mathrm{~d}$ in 1 atmosphere and then in a vacuum for $2 \mathrm{~h}$. The weight of the dry polymer composites was measured and the abovementioned immersion, drying and weighing process for the composite was repeated until it can be considered to be a constant value, $M$. This can be regarded as the dry weight of the composite at its equilibrium state. The dry weight uptake of the polymer composites at equilibrium, $W(\%)$, was determined from the following equation: $W=100\left(M-M_{0}\right) / M$, where $M_{0}$ is the dry weight of the initial polymer complex.

$\mathrm{X}$-Ray powder diffraction patterns of the composites were obtained with the X-ray diffractometer D8 Advance (Bruker AXS, Madison, WI, USA). 


\section{Evaluation of Salt Solution Saturation Level}

To determine the saturation state of the salt solutions with respect to HAP, we introduced a CELLYARD ${ }^{\mathrm{TM}}$ pellet in the solutions as a seed crystal. If deposition took place on the pellet, the solution was deemed to be supersaturated, and if no deposition occurred, the solution was deemed to be undersaturated. We checked the deposition by measuring the weight change $\Delta M$ of the pellet and by visual observation of the pellet surface. Three kinds of solutions, designated as $S, \mathrm{~s}-\mathrm{SBF}$, and $1.5 \mathrm{~s}-\mathrm{SBF}$, were checked for supersaturation: $S$ was a salt solution containing $x \mathrm{mM} \mathrm{CaCl}_{2}, 0.6 x \mathrm{mM} \mathrm{KH}{ }_{2} \mathrm{PO}_{4}(x=5)$, $0.139 \mathrm{mM}$ in the repeating unit of PAA (MW $2000 \mathrm{Da}$ ), and $140 \mathrm{mM} \mathrm{NaCl}$ with Tris- $\mathrm{HCl}$; and $1.5 \mathrm{~s}-\mathrm{SBF}$ was a solution containing $\mathrm{CaCl}_{2}$ and $\mathrm{K}_{2} \mathrm{HPO}_{4}$ at concentrations 1.5 times those of $\mathrm{s}-\mathrm{SBF}$.

\section{RESULTS}

The results of the experiments on the saturation levels of the salt solutions with respect to HAP are listed in Table I.

The s-SBF was supersaturated, as indicated by the weight increase of the pellet $(1.4 \mathrm{mg})$ and the visible deposition on its surface. The $1.5 \mathrm{~s}-\mathrm{SBF}$ solution, which had a higher salt concentration than s-SBF, showed a $17.1 \mathrm{mg}$ increase in weight, which was much larger than that for s-SBF. These results indicate that this method for determining the saturation state was valid. Solution $S$ was determined to be undersaturated with respect to HAP: the weight increases for the pellet immersed in this solution was negligible $(0.2 \mathrm{mg})$, and no deposition was observed on the pellet surface. Solution $S$ has the highest salt concentrations among the solutions we adopt in the following experiments. Hence, it can be concluded that pure HAP cannot be mineralized by those experiments.

When polymer complex films were immersed in salt solutions at various salt concentrations $x(0 \leq x \leq 5)$, they began to swell and were transformed into hydrogels. After the solutions were renewed several times, the composites were rinsed, dried and weighed. This process was repeated until the weight no longer changed. After equilibrium was reached, the weight uptake of the composites was measured in their dry states, and the results are shown in Figure 1.

Four composites ( $x=3.5,3.5,3.65$ and 3.7) were combined and powdered for X-ray diffraction analysis. The XRD pattern is shown in Figure 2, along with the pattern of the composite for $x=5$.

For $x \leq 3.7$, the composites were hydrogels, and the dry weight uptake of the solid component increased gradually with increasing $x$, up to $40 \%$ in dry weight. The X-ray diffraction pattern of the combined composites (Figure 2(a)) shows no

Table I. Saturation levels of salt solutions

\begin{tabular}{ccccc}
\hline Solution & Immersion time $(\mathrm{h})$ & $\boldsymbol{\Delta} M(\mathrm{mg})$ & Surface & Saturation \\
\hline S & 163 & 0.2 & No deposition & Undersaturation \\
s-SBF & 166 & 1.4 & Deposition & Supersaturation \\
$1.5 \mathrm{~s}-\mathrm{SBF}$ & 167 & 17.1 & Deposition & Supersaturation \\
\hline
\end{tabular}

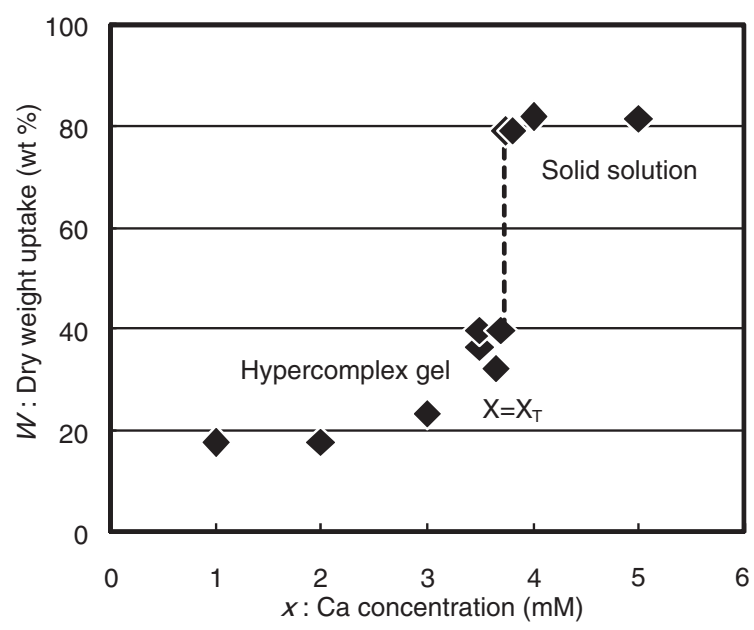

Figure 1. Dry weight uptake by polymer complex films at equilibrium.

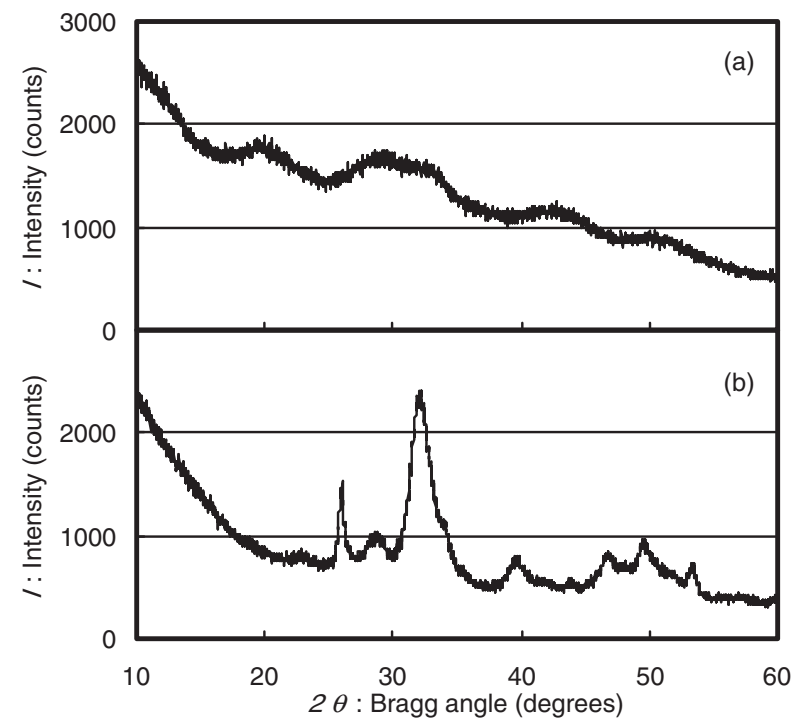

Figure 2. X-Ray powder diffraction patterns of a mixture of composites for $x=3.5,3.5,3.65$ and $3.7(a)$, and the composite $x=5(b)$.

crystallinity. Therefore the weight uptake in this range of $x$ values originated from the absorption of $\mathrm{Ca}^{2+}$ and $\mathrm{HPO}_{4}{ }^{2-}$ ions into the PVA/PAA complex. The composite obtained was a hypercomplex ${ }^{1}$ gel having the cross-linking structure between PVA and PAA depicted in Figure 3.

In the hypercomplex, $\mathrm{Ca}^{2+}$ was absorbed as a counter ion for PAA, and $\mathrm{HPO}_{4}{ }^{2-}$ that was held between PVA and PAA by hydrogen bonding. In this way, the polymer complex was miscible with $\mathrm{Ca}^{2+}, \mathrm{HPO}_{4}{ }^{2-}$ and water, and therefore changed into hypercomplex gel by swelling in the salt solution. It will be difficult to avoid perfectly the leakage of the $\mathrm{HPO}_{4}{ }^{2-}$ trapped between PVA and PAA in rinsing process. The extent of this leakage will be dependent on rinsing motion and gel thickness. This can be the origin of experimental errors appeared in Figure 1.

In contrast, for $3.75 \leq x$, the hydrogel became rigid as time passed. The weight uptake of the solid component was about 


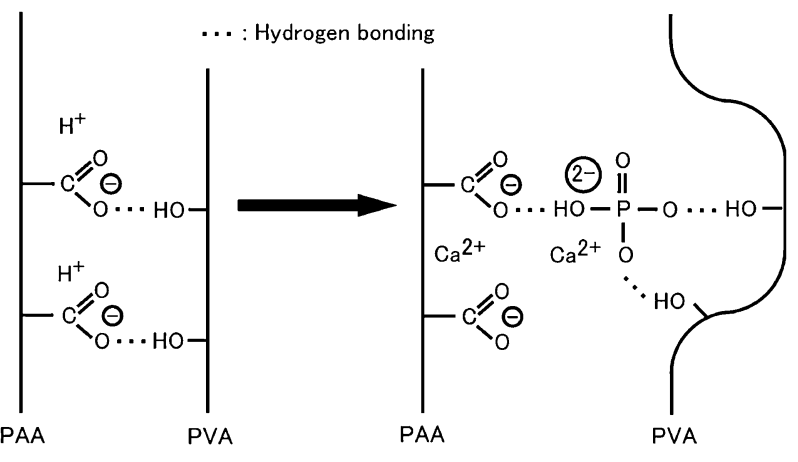

Polymer complex

Hypercomplex

(Model of cartilaginous material)

Figure 3. Structures of the PVA/PAA polymer complex (left) and the hypercomplex obtained after immersion in a salt solution (right).

$80 \mathrm{wt} \%$, and this value was seemed to be independent of $x$ (Figure 1). Because these solid composites absorbed little water, the space between the polymer chains of the network was filled with solid components. ${ }^{1}$ The X-ray powder diffraction pattern of this solid composite (Figure 2(b)) had peaks at the same Bragg angles as those of HAP, although the former peaks were broader than the latter ones. ${ }^{10}$ The XRD pattern indicated that the weight uptake of the solid composite can be ascribed to HAP component.

We can determine the equilibrium state of the solid composite immersed in an undersaturated outer salt solution by examining the equality of chemical potentials of HAP at the interface between solid composite and undersaturated outer salt solution. At equilibrium, the chemical potential of the HAP in the solid composite, $\mu_{\mathrm{IN}}$, is equivalent to the chemical potential of the HAP in the outer salt solution, $\mu_{\text {OUT }}: \mu_{\mathrm{IN}}=\mu_{\text {OUT }}$. Since the outer solution was undersaturated, $\mu_{\text {OUT }}$ is less than the chemical potential of HAP at saturation, $\mu_{\mathrm{SAT}}: \mu_{\mathrm{OUT}}<\mu_{\mathrm{SAT}}$. The $\mu_{\mathrm{SAT}}$ can be represented by the standard chemical potential of (pure) HAP, $\mu_{0}: \mu_{\mathrm{SAT}}=\mu_{0}$. Therefore the inequality, $\mu_{\mathrm{IN}}<\mu_{0}$, is obtained. This is satisfied when $\mu_{\mathrm{IN}}$ is expressed as $\mu_{\mathrm{IN}}=\mu_{0}+R T \ln a$, where $R$ is the gas constant, $T$ is the temperature, and $a(a<1)$ is the activity of HAP in the solid composite. The relation, $a<1$, means that HAP is miscible with the organic polymer complex and therefore the solid composite is a solid solution of organic polymer complex and HAP. In other words, HAP is a good solvent to accomplish the swelling of the polymer network of PVA/PAA complex. In this way, the solid composites form "solid gel phase." The free energy of the solid solution is lower than that of the system in which the HAP and the PVA/PAA network exist separately. Therefore, a solid solution of PVA/PAA and HAP can be formed in a solution undersaturated with respect to HAP. Solid solutions are classified in two categories, that is, substitutional and interstitial solid solutions. For the solid solution of organic polymer and HAP, the model structures corresponding to substitutional and interstitial solid solutions are shown in Figure 4.
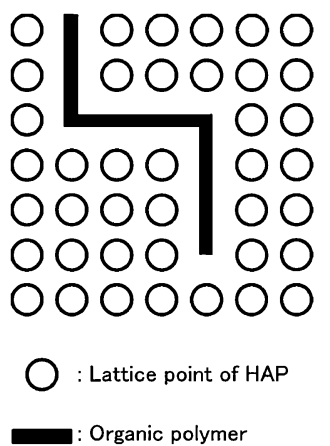

(a)
01000000 0000000 0000000 0000000 0000000 00001000 0000000

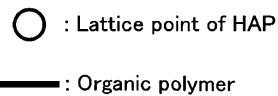

(b)
Figure 4. Schematic illustration of substitutional (a) and interstitial (b) solid solutions of organic polymer and HAP. The three-dimensional hexagonal lattice of HAP is represented as a two-dimensional square lattice.

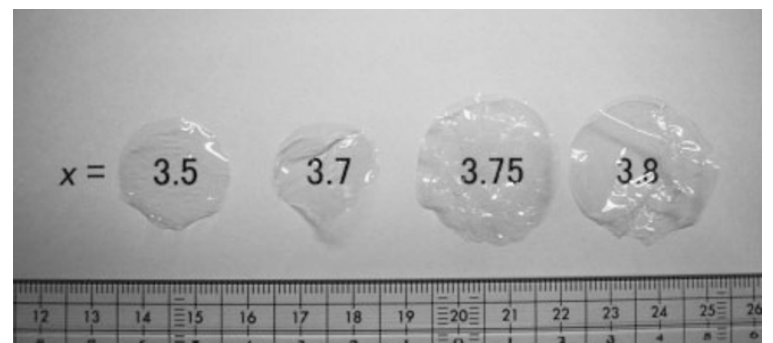

Figure 5. Dried composites after equilibrium at $x=3.5,3.7,3.75$, and 3.8 .

In a substitutional solid solution, the lattice points of HAP are partially replaced with repeating units of organic polymer, and in an interstitial solid solution, the organic polymer fits into the spaces between the HAP lattice points. Figure 4 shows an ideal model structure in which the main lattice points of HAP are not dislocated. However, the presence of the organic polymer will inevitably cause a slight dislocation of the HAP lattice points, no matter which structure the solid solution takes. This slight dislocation affects the X-ray powder diffraction patterns and broadens the peaks located at Bragg angles the same as those of HAP.

Figure 5 shows a photograph of four dried composites equilibrated at $x=3.5,3.7,3.75$, and 3.8, respectively. For $x=3.5$ and 3.7 , the swollen gels shrank after being dried, whereas the solid composites for $x=3.75$ and 3.8 kept their shapes and sizes. The transparency of these composites indicates the miscibility of the components (polymer network and ions for $x<x_{\mathrm{T}}$; polymer network and HAP for $x, x_{\mathrm{T}}<x$ ) of the composites. From the standpoint of thermodynamics, each composite forms a stable phase because of the miscibility of its components.

As we have already mentioned, the equilibrium state of organic/inorganic composite changed discontinuously at $x=$ $x_{\mathrm{T}}\left(3.7<x_{\mathrm{T}}<3.75\right)$ from a hypercomplex gel to an organic/ inorganic solid solution. Owing to the discontinuity, this change should be regarded as a phase transition. This phase transition caused by the increase of $x$ is a transition of an open system and appears to be accompanied by hydrolysis: 
Table II. Proposed phase transitions from hypercomplex gels to organic/inorganic solid solutions for various skeletal materials

\begin{tabular}{cccc}
\hline Skeletal material & $\begin{array}{c}\text { lonic polymer raising } \\
\text { precipitation threshold }\end{array}$ & Absorbed anion & Reaction \\
\hline$\left\{\mathrm{Ca}_{5}\left(\mathrm{PO}_{4}\right)_{3}(\mathrm{OH})\right\}_{\mathrm{SS}}$ & Polyanion & $\mathrm{HPO}_{4}{ }^{2-}$ & Hydrolysis \\
$\left\{\mathrm{CaCO}_{3}\right\}_{\mathrm{SS}}$ & Polyanion & $\mathrm{HCO}_{3}{ }^{-}$ & Weak alkali \\
$\left\{\mathrm{SiO}_{2}\right\}_{\mathrm{SS}}$ & Polycation & $\mathrm{HSiO}_{3}{ }^{-}$ & Hydrolysis \\
Dehydration alkali & Weak acid \\
\hline
\end{tabular}

$$
\left\{\begin{array}{r}
\}+5 \mathrm{Ca}^{2+}+3 \mathrm{HPO}_{4}{ }^{2-}+\mathrm{H}_{2} \mathrm{O} \\
\rightarrow\left\{\mathrm{Ca}_{5}\left(\mathrm{PO}_{4}\right)_{3}(\mathrm{OH})\right\}_{\mathrm{SS}}+4 \mathrm{H}^{+},
\end{array}\right.
$$

where \{\} is the hypercomplex gel and $\left\{\mathrm{Ca}_{5}\left(\mathrm{PO}_{4}\right)_{3}(\mathrm{OH})\right\}_{\mathrm{sS}}$ is the solid solution of the polymer network and $\mathrm{Ca}_{5}\left(\mathrm{PO}_{4}\right)_{3}(\mathrm{OH})$. Comparison of the present system with a usual supersaturated solution of HAP is useful for understanding the phase transition (1). In a supersaturated solution, $\mathrm{Ca}_{5}\left(\mathrm{PO}_{4}\right)_{3}(\mathrm{OH})$ precipitates with hydrolysis:

$$
5 \mathrm{Ca}^{2+}+3 \mathrm{HPO}_{4}{ }^{2-}+\mathrm{H}_{2} \mathrm{O} \rightarrow \mathrm{Ca}_{5}\left(\mathrm{PO}_{4}\right)_{3}(\mathrm{OH})+4 \mathrm{H}^{+}
$$

Consequently the supersaturated solution separates into two phases, a HAP phase and a saturated solution phase (phase separation). In our artificial biomineralization system, instead of phase separation (2), phase transition (1) occurs within the polymer gel immersed in an undersaturated solution because the affinity between the polymer network and HAP is so high that a solid solution of the two can be formed. In this sense, the polymer complex gel is utilized as a mineralization field of HAP.

We accomplished the phase transition from the hypercomplex gel to the organic/inorganic solid solution by varying the $\mathrm{Ca}^{2+}$ and $\mathrm{HPO}_{4}{ }^{2-}$ concentrations, with the constraint that $\left[\mathrm{HPO}_{4}{ }^{2-}\right] /\left[\mathrm{Ca}^{2+}\right]=0.6$. It can be supposed that changing another parameter, such as $\mathrm{pH}, \mathrm{NaCl}$ concentration, or the PVA/PAA ratio of the polymer complex may also cause such a phase transition.

\section{DISCUSSION}

Our model can be applied to various skeletal materials (Table II). Bone contains collagen, acidic proteins and HAP, whereas vertebrate cartilage contains collagen, acidic proteins (proteoglycans), Ca, P and water. ${ }^{13}$ We suggest that HAP-based skeletal material can be considered to be a solid solution of collagen, acidic proteins, and HAP, whereas cartilage can be considered to be a hypercomplex gel composed of collagen, proteoglycans, $\mathrm{Ca}^{2+}, \mathrm{HPO}_{4}{ }^{2-}$, and water. When PVA and PAA are replaced, respectively, with collagen, in which hydroxyproline residues are abundant, and with proteoglycans in the schematic shown in Figure 3, the resulting hypercomplex gel represents the fundamental structure of cartilage. Therefore, our model implies that cartilaginous material may change to HAP-based skeletal material by means of a phase transition analogous to that observed in our artificial biomineralization system.

It seems natural to extend this mechanism to the formation of $\mathrm{CaCO}_{3}$ skeletal materials. At weak alkaline $\mathrm{pH}$ values, a hypercomplex gel will be formed by the absorption of $\mathrm{HCO}_{3}{ }^{-}$ with $\mathrm{Ca}^{2+}$ as a counter ion in the polymer complex mentioned in introduction. Such a hypercomplex gel would be similar in structure to the schematic in Figure 3, except that PVA, PAA, and $\mathrm{HPO}_{4}{ }^{2-}$ are replaced with related biopolymers and $\mathrm{HCO}_{3}{ }^{-}$ respectively. Under appropriate conditions, this hypercomplex gel can be expected to undergo a phase transition accompanied by hydrolysis and change to a solid solution of the polymer complex network and $\mathrm{CaCO}_{3}$ :

$$
\{\quad\}+\mathrm{Ca}^{2+}+\mathrm{HCO}_{3}{ }^{-} \rightarrow\left\{\mathrm{CaCO}_{3}\right\}_{\mathrm{SS}}+\mathrm{H}^{+} .
$$

This scenario can also be extended to the formation of $\mathrm{SiO}_{2}$ skeletal materials. At weakly acidic $\mathrm{pH}$ values, a hypercomplex gel in which $\mathrm{HSiO}_{3}{ }^{-}$is absorbed in organic polymer complex gel with hydrogen bonds can be formed. Under appropriate conditions, this hypercomplex gel can be expected to changes to a solid solution of organic polymer complex and $\mathrm{SiO}_{2}$ by a phase transition accompanied by dehydration:

$$
\{\quad\}+\mathrm{HSiO}_{3}{ }^{-} \rightarrow\left\{\mathrm{SiO}_{2}\right\}_{\mathrm{SS}}+\mathrm{OH}^{-} .
$$

Reaction (4) directly exhibits the important role played by acidic ions in the formation of skeletal materials. In order to raise the precipitation threshold, the polymer dissolved in the solution should be dissociated to decrease the local activity product of dissolved ions. ${ }^{1,4}$ Sillafin-1 is basic and a short chain length polymer. ${ }^{9}$ So it can be dissociated at weakly acidic $\mathrm{pH}$ value and therefore will be a candidate of ionic polymer which raise precipitation threshold of the salt solution in silica biomineralization.

According to our model, all skeletal materials can be thought of as solid solutions in which organic polymers and biominerals are stabilized with affinitive interactions forming a solid gel. This model can be used to explain some phenomena observed for skeletal materials. For usual fiber-reinforced composite materials, the surface of the fiber should have wettability to the matrix material surrounding the fibers. Otherwise, the composite can't be reinforced material because the strong junction between fiber and matrix material can't be formed. In our model, skeletal materials can also be regarded as fiber-reinforced composite materials in which the fibers correspond to individual polymer chains because the polymer chains are miscible with the mineral component, that is, wettable in molecular level. This fiber-reinforced material model can explain why skeletal materials can have rupture resistance. Tooth enamel contains about $96 \mathrm{wt} \%$ HAP and is the hardest tissue in the body. The hardness will be due to a type of solid solution hardening like that observed for steel, which is a solid solution of iron and a small amount of carbon and is harder than iron. Such hardening effect is due to very slight distortion of the lattice of the mother component. ${ }^{14}$ In 
$\mathrm{CaCO}_{3}$ skeletal materials, not only calcite but also alagonite and vaterite structures are found depending on the species of organism and the portion of the skeletal material, though calcite is most stable state among the three structures. In our model, the free energy of solid solution of $\mathrm{CaCO}_{3}$ and organic polymer network is considered to be dependent to the network itself. Therefore, the crystal type which gives lowest energy of the solid solution for a given polymer network can be formed in the solid solution.

Some invertebrates have cartilage. The cartilages found in Annelida, Mollusca and Arthropoda species are essentially made up of collagen and polyanionic glycans. ${ }^{15}$ Squid cartilage contains condroitin polysulfate ${ }^{16}$ and collagen. ${ }^{17,18}$ Some types of squid have $\mathrm{CaCO}_{3}$ skeletal material rather than cartilage; this material, called squid pen, contains collagen, chitin and acidic polymers. ${ }^{19}$ During the regeneration of sea urchin spine, precursor formation has been observed by scanning electron microscopy; the precursor, which contains $\mathrm{Ca}$, carbonate, and water, shows no spots in the electron diffraction pattern, which indicates that it is not crystalline. ${ }^{20}$ The precursor is transformed into the calcite skeletal material of sea urchin spine. In our model, this precursor can be considered to be cartilaginous material rather than amorphous $\mathrm{CaCO}_{3}$ that is intrinsically unstable. These examples imply that the phase transition of cartilaginous material to skeletal material may take place in phylogenetic evolution or ontogenesis in many organisms.

A schematic of the phase transition system is shown in Figure 6. The organic/inorganic composite exists in an undersaturated salt solution containing an ionic polymer (not shown) which dissociates and therefore raises precipitation threshold. This composite is an open system, the border of which is permeable to both energy and mass (ions and water). When $x<x_{\mathrm{T}}$, the hypercomplex gel is the thermodynamically stable phase of the organic/inorganic composite. However, when $x_{\mathrm{T}}<x$, the hypercomplex gel becomes unstable and changes to an organic/inorganic solid solution by means of a phase transition accompanied by hydrolysis or dehydration. In this region of $x$, the solid solution is the thermodynamically stable phase of the organic/inorganic composite. The reversibility of this transition will be investigated in future work.

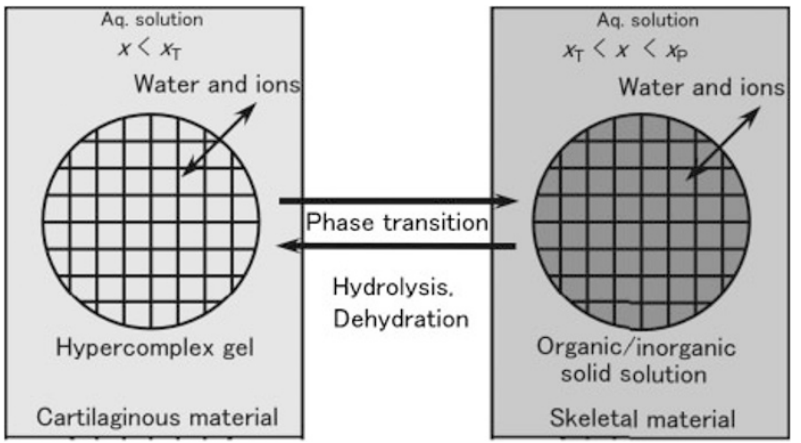

Figure 6. Schematic illustration of the phase transition of an open system between a hypercomplex gel phase (left) and an organic/inorganic solid solution phase (right). $x_{\mathrm{P}}$ is the salt concentration at which precipitation occurred. The equilibrium state changes discontinuously at the transition concentration $x=x_{\mathrm{T}}$.

\section{CONCLUSION}

In our artificial biomineralization system, a hypercomplex gel and an organic/inorganic solid solution can be defined as thermodynamic phases because their constituents are miscible in each composite. The hypercomplex gel changed to an organic/inorganic solid solution by means of a phase transition. Therefore the hypercomplex gel and the organic/ inorganic solid solution are the two phases of an open organic/inorganic composite system. This model seems to be applicable not only to the formation of solid solutions of organic polymers and HAP but also to the formation of solid solutions of organic polymers and $\mathrm{CaCO}_{3}$ and $\mathrm{SiO}_{2}$. We suggest that the hypercomplex gel can serve as a model for cartilaginous material and that the organic/inorganic solid solution can serve as a model for skeletal material. From our results, we can deduce that under appropriate conditions (salt concentrations, and probably polymer density, polymer ratio, $\mathrm{pH}$, etc.), cartilaginous material can be converted to skeletal material by means of a phase transition in biological system. Thus we have used our model to describe three types of skeletal materials and their corresponding cartilaginous materials within a common framework.

Acknowledgment. We thank Ms. Jahan Akther who kindly checked the manuscript. We gratefully acknowledge the financial support provided by Grant-in-Aid for Scientific Research of the Ministry of Education, Culture, Sports, Science, and Technology (MEXT) of Japan (B-17350113; 2005).

Received: May 23, 2008

Accepted: June 30, 2008

Published: August 12, 2008

\section{REFERENCES}

1. T. Iwatsubo, K. Sumaru, T. Kanamori, T. Shinbo, and T. Yamaguchi, Biomacromolecules, 7, 95 (2006).

2. M. R. Urist, A. Mikulski, and A. Lietze, Proc. Natl. Acad. Sci. U.S.A., 76(4), 1828 (1979).

3. G. K. Hunter, P. V. Hauschka, A. R. Poole, L. C. Rosenberg, and H. A. Goldberg, Biochem. J., 317, 59 (1996).

4. M. Nagasawa and I. Kagawa, J. Polym. Sci., XXV, 61 (1957).

5. H. Endo, Y. Takagi, N. Ozaki, T. Kogure, and T. Watanabe, Biochem. J., 385, 159 (2004).

6. H. Inoue, N. Ozaki, and H. Nagasawa, Biosci., Biotechnol., Biochem., 65(8), 1840 (2001).

7. N. Kröger, C. Bergsdorf, and M. Sumper, EMBO J., 13(19), 4676 (1994).

8. N. Poulsen, M. Sumper, and N. Kröger, Proc. Natl. Acad. Sci. U.S.A., 100, 12075 (2003).

9. N. Kröger, R. Deutzmann, and M. Sumper, Science, 286, 1129 (1999).

10. T. Iwatsubo, S. P. Kusmocahyo, T. Kanamori, and T. Shinbo, J. Appl. Polym. Sci., 100(2), 1465 (2006).

11. P. W. Kuhn, B. Hargitay, A. Katchalsky, and H. Eisenberg, Nature, 165, 514 (1950).

12. A. Oyane, K. Onuma, A. Ito, H.-M. Kim, T. Kokubo, and T. 
Nakamura, J. Biomed. Mater. Res., 64A(2), 339 (2002).

13. M. A. Logan, J. Biol. Chem., 110, 375 (1935).

14. P. Feltham, J. Phys. D: Appl. Phys., 1, 303 (1968).

15. P. Person and D. E. Philpott, Biol. Rev. Camb. Philos. Soc., 44, 1 (1969).

16. Y. Kawai, N. Seno, and K. Anno, J. Biochem., 60(3), 317 (1966).

17. P. Sivakumar and G. Chandrakasan, Biochim. Biophys. Acta, 1381,
161 (1998).

18. S. Kimura and K. Karasawa, Comp. Biochem. Physiol., 81B(2), 361 (1985).

19. S.-D. Wu, C.-S. Wu, and H.-C. Chen, Fish. Sci., 69, 849 (2003).

20. Y. Politi, T. Arad, E. Klein, S. Weiner, and L. Addadi, Science, 306, 1161 (2004). 